\title{
Delayed diagnosis in retinoblastoma
}

\author{
Karla E. S. Rodrigues, ${ }^{1}$ Maria do Rosário D. O. Latorre, ${ }^{2}$ Beatriz de Camargo ${ }^{3}$
}

\begin{abstract}
Objectives: To identify the main symptoms of retinoblastoma and to determine the mean time between symptom onset and diagnosis (lag time).

Patients and methods: We carried out a retrospective analysis of the patients diagnosed with retinoblastoma between January 1991 and June 2000, at the Pediatric Department of the Hospital do Câncer, São Paulo, Brazil. Statistical analyses performed were: Student's $t$ test, ANOVA, Tukey-HSD test (honest significant differences), Levene's test, multiple regression, ROC curve, logistic regression, Kaplan-Meier, and log rank.

Results: 327 medical records (171 males) were reviewed. The mean age was 25 months. Localized disease was identified in 269 patients. The most frequent symptoms were leukocoria (79\%), strabismus $(10.7 \%)$ and tumor mass (3.4\%). Mean lag time was 5.8 months. Patients older than 2 years old had longer lag time in comparison to infants ( 7.2 vs 4.7 months; $\mathrm{p}=0.001)$. Lag time was longer among patients with strabismus ( 8.8 months) in comparison to patients with tumor mass ( 2.3 months) and leukocoria ( 5.6 months); $\mathrm{p}=0.014$. Patients with metastatic disease had longer lag time $(10.6$ months; $p<0.001)$. Lag time was influenced by advanced disease $(O R=3.25 / C I=$ $1.61: 6.55)$, metastatic disease $(\mathrm{OR}=3.52 / \mathrm{CI}=1.21: 10.21)$ and strabismus $(\mathrm{OR}=2.84 / \mathrm{IC}=1.36: 5.92)$. Five-year overall survival rates were higher among patients with localized disease $(94.6 \%)$ and among patients with lag time less than 6 months $(91 \%)$, in comparison to patients with longer lag time $(78 \% ; p<0.001)$.

Conclusion: Most frequent symptoms of retinoblastoma were leukocoria, strabismus and tumor mass. Mean lag time was 5.8 months. Strabismus and advanced disease were associated to longer lag time. Patients with advanced
\end{abstract} disease and lag time longer than 6 months had worse prognosis.

J Pediatr (Rio J). 2004;80(6):511-6: Retinoblastoma, delay of diagnosis, signs and symptoms, leukocoria, strabismus.

\section{Introduction}

Retinoblastoma is the most common intraocular malignancy of childhood. It affects one in every 20,000 live births in the United States. ${ }^{1}$ In Brazil, in the city of São Paulo, between 1969 and 1997/98, the average incidence rates were $8.7 /$ million boys and 8.1 /million girls. ${ }^{2}$ Although retinoblastoma accounts for approximately $3 \%$ of pediatric

1. M.Sc. Pediatric oncologist. Fundação Antônio Prudente, Center of Treatment and Research, Hospital do Câncer de São Paulo, São Paulo, SP, Brazil.

2. Department of Epidemiology, Faculdade de Saúde Pública, Universidade de São Paulo (USP), São Paulo, SP, Brazil.

3. Pediatric oncologist. Chief of the Department of Pediatrics, Center of Treatment and Research, Hospital do Câncer de São Paulo, São Paulo, SP, Brazil.

Financial support: FAPESP.

Manuscript received Apr 22 2004, accepted for publication Sep 082004.

Suggested citation: Rodrigues KE, Latorre MR, de Camargo B. Delayed diagnosis in retinoblastoma. J Pediatr (Rio J). 2004;80:511-6. malignancies in industrialized countries, there is some evidence that this tumor is more frequent in developing countries of Latin America, Africa and India. ${ }^{3}$ In these countries, retinoblastoma often is diagnosed too late, when the disease already shows extraocular dissemination, thus resulting in a poor prognosis. ${ }^{4}$ Successful treatment of retinoblastoma depends on the ability of parents and pediatrician to detect the disease while it is still confined to the eye, promptly referring their children to an ophthalmologist for funduscopic examination and to the pediatric oncologist for adequate treatment according to the stage of the disease, in order to preserve life and useful vision.

The signs and symptoms of retinoblastoma depend on its size and location, but the most common presenting sign is leukocoria, known as cat's eye reflex. Other manifestations include strabismus, conjunctival hyperemia, blindness and glaucoma. When the tumor spreads extraocularly it is 
usually characterized by an orbital mass with proptosis, and when metastasis to the central nervous system is present, headache, vomiting, anorexia and irritability may occur. ${ }^{5}$

Despite the efforts made by physicians and volunteers to increase the early detection of cancer through campaigns that warn against signs and symptoms and through education programs for health professionals, studies have shown little improvement in the time between symptom onset and medical assistance, and also in the rapid establishment of a diagnosis and initiation of proper treatment. ${ }^{6}$

The aim of the present study was to identify the major signs and symptoms of retinoblastoma in our setting, determining the average duration of the symptoms as well as the factors associated with their duration.

\section{Patients and methods}

A retrospective cohort study was carried out at the Department of Pediatrics of Hospital do Câncer de São Paulo, between January 1991 and June 2000. The data were collected from medical charts by one of the researchers (KESR), and a specific database was constructed for the study. The study population consisted of patients diagnosed with retinoblastoma admitted to the afore-mentioned department. The study protocol was approved by the Ethics Committee of the hospital. All medical charts contained the necessary data, so there was no loss. The final sample comprised 327 patients.

The stage of the disease at diagnosis was assessed using the Reese-Ellsworth ${ }^{7}$ classification system for intraocular tumors and the classification proposed by the Children's Cancer Study Group (CCSG-962) ${ }^{8}$ for extraocular tumors. The analysis considered localized disease (intraocular), advanced disease (extraocular, classes I, II and III) and metastatic disease (extraocular, classes IV and V).

The comparison between the mean duration of the symptoms and the various studied factors was made using Mann-Whitney test (when the variable had two categories) and Kruskal-Wallis test (when the variable had three or more categories). The multiple comparisons were made using the Tukey's honest significant differences test (Tukey's HSD) and Levene's test for homogeneity of variance. The multiple factor analysis was carried out by linear regression analysis, where the duration of the symptoms was the dependent variable and the other characteristics (age, gender, race and extent of the disease) were the independent variables. ${ }^{9}$ In the multiple regression analyses, coefficient values, their respective $p$ values, and the adjusted coefficients of determination ( $\operatorname{adj} r^{2}$ ) were calculated. The latter coefficients range from zero to one and represent the explanatory power of the model - the closer to one, the better the explanatory power of the model.

A second analysis was made to assess the duration of the symptoms as a dichotomous variable. By using an ROC curve, we determined the cutoff point for the duration of symptoms that obtained the best sensitivity (51\%) and specificity $(76 \%)$ for the occurrence of death (Figure 1$).{ }^{10}$ Based on that, the delay variable was developed, and no delay $(Y=0)$ was considered when the duration of symptoms was up to 6 months, and delay $(Y=1)$ was when the duration of symptoms was longer than six months. Univariate and multiple logistic regression analyses were made using the delay as a dependent variable. The significance of the model was assessed by Hosmer \& Lemeshow test. ${ }^{10}$ The stage of the disease and delayed diagnosis were regarded as prognostic factors for death, using the Kaplan \& Meier product limit estimator. ${ }^{11}$ The curves were compared using the log-rank test. The SPSS statistical package for Windows, version 10.0, was used for all statistical analyses and the significance level was established at $5 \%$.

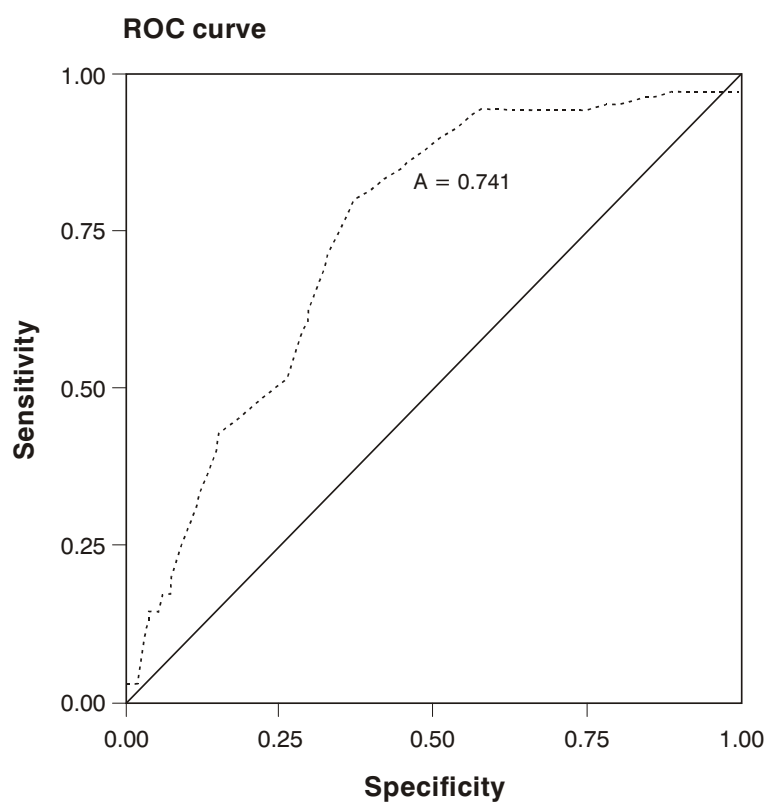

Figure 1 - ROC curve of duration of symptoms

\section{Results}

Out of 327 patients, 171 (52.3\%) were male and 156 $(47.7 \%)$ were female, with a $1.1: 1$ male/female ratio. There was predominance of white patients $(80.4 \%)$. Age ranged from two months to twelve years, mean of 25 months (standard deviation $=19.5$ months and median of 22.2 months). Fifty-three percent were infants ( 0 to 24 months), followed by preschoolers (44\%), and only nine patients were older than 6 years.

At diagnosis, $82.2 \%$ of the patients had localized disease, $12.8 \%$ showed advanced disease and $5 \%$ metastatic disease. The most common manifestations were leukocoria (79\%), strabismus (10.7\%) and tumor growth (3.4\%).

The duration of symptoms ranged from 3 days to 48 months, mean of 5.8 months (standard deviation $=6.6$ months) and median of 3 months. No statistically significant 
differences were observed between average durations according to gender and complexion (Table 1). Patients aged 24 months or older had a longer duration of symptoms than did infants ( 7.2 months versus 4.7 months; $p=0.001$ ). Patients with strabismus had a longer average duration of symptoms (8.8 months), when compared to those whose symptoms were tumor growth (2.3 months) or leukocoria (5.6 months). Patients with localized disease showed a shorter duration of symptoms (4.8 months) than those with advanced disease ( 9.3 months) or with metastatic disease (10.6 months).

Table 2 presents the linear regression model, where duration of symptoms was a dependent variable. Despite the low explanatory power of the model (adj $\left.r^{2}=0.065\right)$, the significant independent determinants for the duration of symptoms were the presence of strabismus and advanced disease The variables age and complexion were maintained in the multiple model as control variables and were of little significance ( $p=0.061$ and $p=0.083$, respectively). In case of strabismus, the duration of symptoms increased by 0.233 months, regardless of age, complexion and stage of the disease. Patients with advanced disease showed on average a delay of 0.181 months more, regardless of age, complexion and presence of strabismus.

As described in the methodology for the logistic regression analysis, we used a cutoff point of 6 months for the duration of symptoms, which was established using an ROC curve
Table 2 - Simple (crude analysis) and multiple (adjusted analysis) linear regression model of duration of symptoms

\begin{tabular}{lcccc}
\hline Characteristic & \multicolumn{2}{c}{ Crude analysis } & \multicolumn{2}{c}{ Adjusted analysis } \\
& Coefficient & $\mathbf{p}$ & Coefficient & $\mathbf{p}$ \\
\hline Age & 7.444 & $<0.001$ & 2.439 & 0.061 \\
Race & -3.440 & $<0.001$ & -0.109 & 0.083 \\
Strabismus & 3.355 & 0.005 & 0.233 & 0.003 \\
Advanced disease & 5.511 & $<0.001$ & 0.181 & 0.018
\end{tabular}

$r^{2} a j=0.065 ; p($ model $)<0.001$.

(Figure 1). Independent factors associated with delayed diagnosis were the presence of advanced disease (OR = $3.25)$, presence of metastatic disease $(O R=3.52)$ and presence of strabismus $(O R=2.84)$ (Table 3$)$.

Figure 2 shows that patients with metastatic and advanced disease had a worse prognosis than those with localized disease (chances of five-year survival of 0.60 and $94.6 \%$, respectively; $\mathrm{p}<0.001$ ). The delayed diagnosis also indicated worse prognosis, the five-year survival rate corresponded to $78 \%$ when the duration of symptoms was longer than six months and to $91 \%$ when the duration of symptoms did not exceed 6 months, $\mathrm{p}<0.001$ (Figure 3).

Table 1 - Mean duration of symptoms (months) according to age, sex, race, symptoms and severity of the disease at diagnosis

\begin{tabular}{|c|c|c|c|c|c|}
\hline Variable & $\mathbf{n}$ & $\%$ & $\begin{array}{c}\text { Mean duration } \\
\text { (months) }\end{array}$ & $\begin{array}{c}\text { DP } \\
\text { (months) }\end{array}$ & $\mathbf{p}$ \\
\hline Age (months) & & & & & 0,001 \\
\hline $0-23.9$ & 174 & 53.2 & 4.7 & 4.6 & \\
\hline$\geq 24$ & 153 & 46.8 & 7.2 & 8.1 & \\
\hline \multicolumn{6}{|l|}{ Sex } \\
\hline Male & 171 & 52.3 & 5.8 & 6.7 & 0.949 \\
\hline Female & 156 & 47.7 & 5.8 & 6.6 & \\
\hline \multicolumn{6}{|l|}{ Race } \\
\hline Caucasian & 263 & 80.4 & 5.1 & 5.6 & 0.533 \\
\hline Non-Caucasian & 64 & 19.6 & 6 & 5.7 & \\
\hline \multicolumn{6}{|l|}{ Symptom } \\
\hline Leukocoria (a) & 258 & 78.9 & 5.6 & 6.5 & 0.014 \\
\hline Strabismus (b) & 35 & 10.7 & 8.8 & 7.2 & $\left({ }^{\#} \mathrm{a}<\mathrm{b} ; \mathrm{p}=0.030\right)$ \\
\hline Tumor (c) & 11 & 3.4 & 2.3 & 3.4 & $\left({ }^{\#} \mathrm{c}<\mathrm{b} ; \mathrm{p}=0.022\right)$ \\
\hline Other * (d) & 23 & 7 & 5.8 & 6.2 & \\
\hline \multicolumn{6}{|l|}{ Severity } \\
\hline Localized (e) & 269 & 82.2 & 4.8 & 5.4 & $<0.001$ \\
\hline Advanced (f) & 42 & 12.8 & 9.3 & 9.2 & $\left({ }^{\#} \mathrm{e}<\mathrm{f} ; \mathrm{p}<0.001\right)$ \\
\hline Metastatic (g) & 16 & 5 & 10.6 & 9.6 & $\left({ }^{\#} \mathrm{e}<\mathrm{g} ; \mathrm{p}=0.020\right)$ \\
\hline Total & 327 & 100 & 5.8 & 6.6 & \\
\hline
\end{tabular}

SD = standard deviation

\# Multiple comparisions (Tukey-HSD).

* Other = anorexia (1), apathy (3), growth delay (3), blindness (3), fever (1), ocular hyperemia (8), nystagmus (1) and eye secretion (3). 
Table 3 - Multiple logistic regression model of delayed diagnosis

\begin{tabular}{lcc}
\hline Variable & Adjusted OR & 95\% CI (ORaj) \\
\hline Advanced disease & 3.25 & {$[1.61: 6.55]$} \\
Metastatic disease & 3.52 & {$[1.21: 10.21]$} \\
Strabismus & 2.84 & {$[1.36: 5.92]$}
\end{tabular}

$\mathrm{p}$ (Hosmer-Lemeshow's test $)=0.981$.

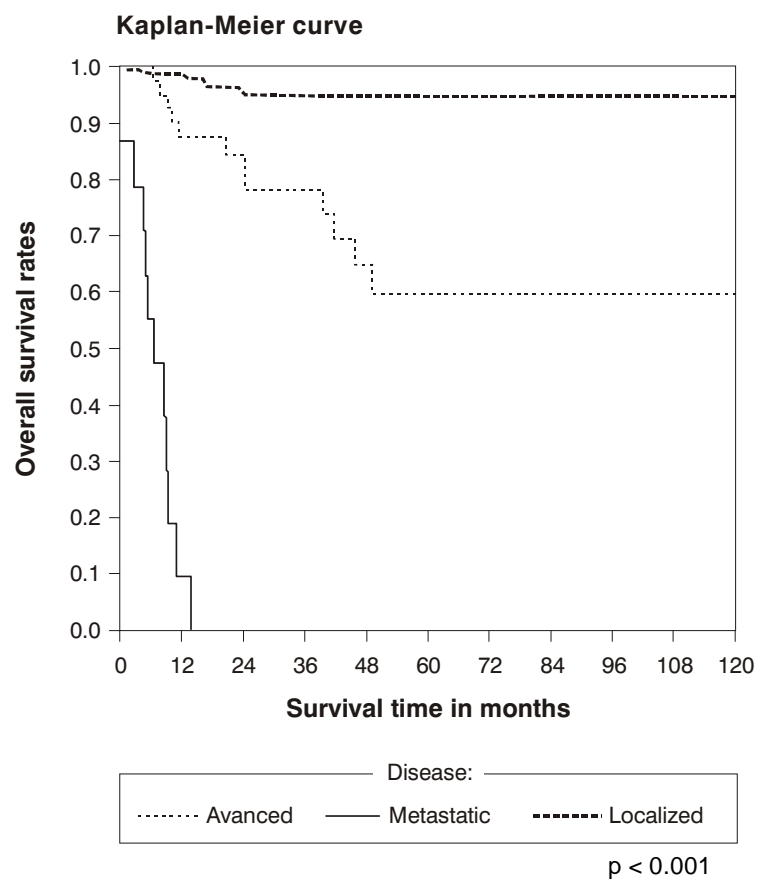

Figure 2 - Survival curve in 5 years according to the severity of the disease at diagnosis

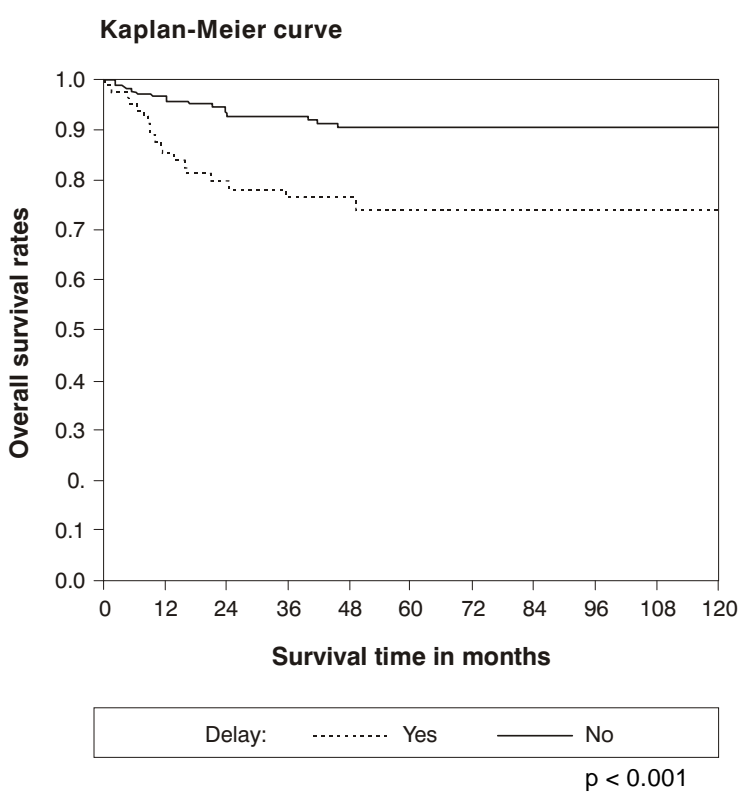

Figure 3 - Survival curve in 5 years according to the duration of symptoms

\section{Discussion}

Between 1953 and 1962, retinoblastoma was the most frequent solid tumor diagnosed at the Department of Pediatrics of Hospital do Câncer de São Paulo, accounting for $19 \%$ of pediatric malignancies. Between 1988 and 1994, retinoblastoma represented $11.1 \%$ of all pediatric malignancies, ranking as the second most frequent solid tumor. This pattern persisted between 1991 and 1997, when it corresponded to the third most common type of tumor among patients admitted to the Department of Pediatrics of that hospital, comprising one of the largest patient populations in Brazil and in Latin America.12,13 Hospital do Câncer de São Paulo is a specialty center for the treatment of retinoblastoma in Brazil; with an interdisciplinary team for the proper treatment of this tumor, including medical specialties such as pediatric oncology, ophthalmology (laser therapy and cryotherapy), external beam radiation therapy and brachytherapy, which are crucial for the treatment of advanced-stage disease and for eye salvage when the tumor is diagnosed at an early stage.

In our study, the mean age of patients was 25 months, being consistent with the literature, where approximately $80 \%$ of the cases are diagnosed before the age of three or four years, on average at 24 months. ${ }^{3}$

Pediatricians play a key role in the diagnosis of ocular tumors, as they often are the first ones to detect ocular disorders, which most times are not perceived by parents. Recognizing the signs and symptoms and referring the patient as soon as possible to funduscopic examination and treatment, if necessary, is of paramount importance. ${ }^{14}$ Pediatricians should also inform and instruct parents about ophthalmic disorders such as strabismus and leukocoria. In a normal eye, if light is shone directly into the pupil, the pupillary space will appear as a homogeneous bright reddish orange color, known as red reflex. It is a reflection of the choroidal vasculature and retinal pigmentation. The signs and symptoms of retinoblastoma depend on its size and location. Leukocoria, which is a white mass overlying or infiltrating the retina, interrupts the red reflex when light is shone into the eye. It is also known as white pupillary reflex or cat's eye reflex. The best way to verify whether leukocoria is present is by direct ophthalmoscopy; however, it can be difficult to visualize in newborns or in children with small pupils. Pupillary dilation can therefore be crucial in detecting small lesions. Canzano \& Handa showed that only $30 \%$ of retinoblastoma cases are detected by direct ophthalmoscopy on undilated examination, whereas $100 \%$ of cases are diagnosed after pupillary dilation. ${ }^{15,16}$ By analyzing the delayed diagnosis of retinoblastoma, Butros et al. noted that parents perceive the first sign/symptom in most cases $(75 \%)$. In $30 \%$ of cases parents seek medical help immediately, but primary care physicians do not refer the patient to a specialist right away. The authors conclude that infants or children with leukocoria must always be referred for ophthalmologic examination regardless of whether an absent red reflex is observed. ${ }^{16}$

Studies conducted in the USA, the UK, Switzerland and Finland stated that leukocoria is the most frequent presenting symptom, found in approximately $50-60 \%$ of cases, followed 
by strabismus (25\%) and inflammatory signs (6-10\%). $17-20$ In this study, $79 \%$ of infants and children presented with leukocoria, followed by strabismus (10.7\%). Other manifestations include conjunctival hyperemia, blindness and glaucoma, and when the tumor spreads extraocularly it is often characterized by an orbital mass with proptosis, and when metastasis to the central nervous system is present, headache, vomiting, anorexia and irritability may occur. ${ }^{5}$ In our study, only 34 out of 327 patients (10.3\%) had manifestations other than leukocoria and strabismus: anorexia, apathy, growth delay, blindness, fever, ocular hyperemia, nystagmus and eye secretion.

The average duration of symptoms was 8.3 months and delayed diagnosis (duration of symptoms longer than six months) was a prognostic factor for poor survival. Antoneli, ${ }^{21}$ by reviewing cases of children with retinoblastoma at the same hospital, demonstrated that the average duration of symptoms in these patients decreased from 7.5 months (1986-1990) to 5.3 months (1991-1995). Sixty-eight percent of these patients showed a duration of symptoms shorter than six months and had a higher rate of intraocular tumor compared to those who had a duration of symptoms longer than six months.

The recommendation that an infant/child with strabismus should be referred to an ophthalmologist early on is not easy to be followed. Approximately 3 to $4 \%$ of children show some degree of strabismus in the first years of life and most children do not show any disorder at all. Strabismus still does not seem to require investigation, a fact that has already been observed in industrialized countries, as in the study conducted by Goddard et al., 22 who found out that although $50 \%$ of children had been referred to an ophthalmologist in the first week after seeing a health agent, $25 \%$ had to wait more than eight weeks. In this study, the risk of delayed diagnosis was higher among younger children with strabismus who had seen a health agent before seeking a clinician. Strabismus is described in nearly $23.6 \%$ of cases, but in our study it was observed in only $10.7 \%$. In case of strabismus, the average duration of the symptom was 8.8 months, which is longer than other symptoms such as leukocoria (5.6 months) or tumor growth (2.3 months). On top of that, strabismus was associated with longer duration of the symptom regardless of age, complexion, and stage of the disease. This shows that maybe this symptom has been underestimated in our setting. ${ }^{17-20}$

There was a predominance of patients with intraocular disease, which shows some improvement in early diagnosis in relation to another study conducted by Erwenne \& Franco ${ }^{4}$ at the Hospital do Câncer de São Paulo. They assessed 153 consecutive patients with retinoblastoma, admitted between 1975 and 1985 and found out that approximately $50 \%$ of the patients presented with extraocular disease at diagnosis and that the parents of these children had underestimated the presence of leukocoria in their children. This may be due to a campaign launched in magazines, newspapers and billboards in São Paulo in 1986, which focused on the early diagnosis of retinoblastoma. In our study, patients with localized disease showed shorter duration of the symptoms compared to those with advanced or metastatic disease. These data confirm the results previously obtained by Erwenne \& Franco $^{4}$ and Antoneli ${ }^{21}$ in our setting, and by Chantada et al. 23 in Argentina. In the Argentinean study, the patients with extraocular disease exhibited symptoms for over six months, and both pediatricians and ophthalmologists failed to make an early diagnosis of retinoblastoma. Out of 56 children with retinoblastoma who first sought a pediatrician, 30 were promptly referred to an ophthalmologist, 26 had their symptoms underestimated and the family decided to see another pediatrician or ophthalmologist. Among 39 patients who first sought an ophthalmologist, the diagnosis was readily made in 30 cases, whereas the remaining nine cases were only diagnosed after consultation with another ophthalmologist. Patients who did not have private health assistance, who lived in the countryside and whose parents had a low educational level showed a more advanced disease. This study shows the influence of continuing education programs for physicians, access to health services and to information by the lay population over delayed diagnosis.

The early diagnosis of retinoblastoma is still a challenge in industrialized and in developing countries, ${ }^{17-20}$ and data recently published by Abramson et al. ${ }^{24}$ suggest that abnormal ocular reflex has not been investigated as recommended. The authors suggest that every infant/ child with family history of retinoblastoma be immediately referred to a pediatric oncologist, and that every newborn infant and child with signs and symptoms such as leukocoria, strabismus, conjunctival hyperemia, proptosis, decrease in visual acuity be submitted to funduscopic examination through a dilated pupil. In addition, education programs for pediatricians, geneticists, obstetricians and ophthalmologists should be offered in order to increase the index of suspicion of retinoblastoma.

Based on these facts, there is a necessity to train health professionals and inform the lay population so that they can identify the initial symptoms of retinoblastoma, especially strabismus. This may result in a larger number of cases with an early diagnosis, thus reducing the mortality of this disease.

Our conclusion is that the most frequent signs and symptoms of retinoblastoma in our setting were leukocoria, strabismus and tumor growth. The average duration of these symptoms is 5.8 months. Strabismus and advanced disease were associated with a longer duration of symptoms. Patients with advanced disease and with a longer duration of symptoms had the worst prognosis.

\section{Acknowledgments}

The authors thank FAPESP (State of São Paulo Research Foundation). Researchers KESR and BC thank the Graduate Program of Antônio Prudente Foundation. Professor MRDOL thanks CNPq for the research grant. 


\section{References}

1. Pendergrass TW, Davis S. Incidence of retinoblastoma in the United States. Arch Ophthalmol. 1980;98:1204-10.

2. Mirra AP, Latorre MRDO, Veneziano DB. Incidência, Mortalidade e Sobrevida do Câncer da Infância no Município de São Paulo. São Paulo: Registro de Câncer de São Paulo; 2004.

3. Hurwitz RL, Shields $C L$, Shields $J A$, Chévez-Barrios $P$, Hurwitz MY, Chintagumpala MM. Retinoblastoma. In: Pizzo PA, Poplack DG, editors. Principles and Practice of Pediatric Oncology. 4th ed. Philadelphia: Lippincont-Raven; 2003. p. 825-46.

4. Erwenne CM, Franco EL. Age and lateness of referral as determinants of extra-ocular retinoblastoma. Ophthalmic Paediatr Genet. 1989;10:179-84.

5. Mackay CJ, Abramson DH, Ellsworth RM. Metastatic patterns of retinoblastoma. Arch Ophthalmol. 1984;102:391-6.

6. Kutner B, Makover H, Opphenheim A. Delay in the diagnosis and treatment of cancer: a critical analysis of the literature. J Chronic Dis. 1958;7:95-120.

7. Reese AB. Tumors of the eye. 3rd ed. Maryland: Harper \& Row; 1976. p. 90-124.

8. Wolff JA, Boesel C, Ellsworth R. Extraocular retinoblastoma. (Children's Cancer Study Group - Protocol CCSG-962). New York; 1978.

9. Dawson-Sanders B, Trapp RG. Basic \& Clinical Bistatistics. 3rd ed. New York: Lange Medical Books/McGraw-Hill; 2001.

10. Hosmer DW, Lemeshow S. Applied logistic regression. New York: John Wiley and Sons; 1989.

11. Kaplan EL, Meier P. Nonparametric estimation from incomplete observations. J Am Stat Assoc. 1958;53:457-81.

12. Ribeiro KCB, de Camargo $B$, Torloni H. Registro Hospitalar de Câncer Pediátrico. 1988 e 1994. Centro de Tratamento e Pesquisa Hospital do Câncer. no. 2, 1999.

13. Mendes WL. Análise das características clínico-epidemiológicas e de sobrevida dos casos de câncer pediátrico do Centro de Tratamento e Pesquisa Hospital do Câncer de São Paulo, $1988,1991,1994$ \& 1997 [dissertação]. São Paulo (SP): Fundação Antonio Prudente; 2002.

14. Castillo BV Jr, Kaufman L. Pediatric tumors of the eye and orbit. Pediatr Clin North Am. 2003;50:149-72.

15. Canzano JC, Handa JT. Utility of pupillary dilation for detecting leukocoria in patients with retinoblastoma. Pediatrics. 1999; 104:e44.
16. Butros LJ, Abramson DH, Dunkel IJ. Delayed diagnosis of retinoblastoma: analysis of degree, cause, and potential consequences. Pediatrics. 2002;109:e45.

17. Abramson DH, Frank CM, Susman M, Whalen MP, Dunkel IJ, Boyd NW. Presenting signs of retinoblastoma. J Pediatr. 1998;132:505-8.

18. Tarkkanen A, Tuovinen E. Retinoblastoma in Finland 19121964. Acta Ophthalmol (Copenh). 1971;49:293-300.

19. Bedford MA, Bedotto C, Macfaul PA. Retinoblastoma. A study of 139 cases. Br J Ophthalmol. 1971;55:19-27.

20. Balmer A, Gailloud C. Retinoblastoma: diagnosis and treatment. Including a clinical study. In: Straub W, editor.Turning points in cataract formation, syndromes, and retinoblastoma. New York: Karger; 1983. p. 36-96.

21. Antoneli CBG. Retinoblastoma: análise da evolução clínica de pacientes portadores de retinoblastoma submetidos a tratamento multidisciplinar [tese]. São Paulo: Universidade de São Paulo; 1999.

22. Goddard AG, Kingston JE, Hungerford JL. Delay in diagnosis of retinoblastoma: risk factors and treatment outcome. $\mathrm{Br} \mathrm{J}$ Ophthalmol. 1999;83:1320-3.

23. Chantada G, Fandino A, Manzitti J, Urrutia L, Schvartzman E. Late diagnosis of retinoblastoma in a developing country. Arch Dis Child. 1999;80:171-4.

24. Abramson DH, Beaverson $K$, Sangani P, Vora RA, Lee TC, Hochberg HM, et al. Screening for retinoblastoma: presenting signs as prognosticators of patient and ocular survival. Pediatrics. 2003; $112(6$ Pt 1):1248-55.

Corresponding author:

Karla Emília de Sá Rodrigues

Rua Bernardo Guimarães, 3106, Barro Preto

CEP 30140-083 - Belo Horizonte, MG

Brazil

E-mail: karla.emilia@terra.com.br 